\title{
TNFa-TNFR2 signaling pathway in control of the neural stem/progenitor cell immunosuppressive effect: Different experimental approaches to assess this hypothetical mechanism behind their immunological function
}

Sara Shamdani ${ }^{1,2}$, Georges Uzan ${ }^{1,3+}$ and Sina Naserian ${ }^{1,2,3^{*+}}$ (i)

\begin{abstract}
Background: Stem cells have a vast range of functions from tissue regeneration to immunoregulation. They have the ability to modulate immune responses and change the progression of different inflammatory and autoimmune disorders. Tumor cells share many characteristics of stem/progenitor cells too. Both can inhibit effector $T$ cells and other immune cells, while inducing regulatory $T$ cells ( $T$ regs), thus, reducing the production of pro-inflammatory cytokines and increasing the production of anti-inflammatory ones. In this context, some cytokines like TNFa are able to control the direction of the immune response. TNF-TNFR signaling plays a dual role: while the interaction of TNFa with TNFR1 mediates pro-inflammatory effects and cell death, its interaction with TNFR2 mediates antiinflammatory effects and cell survival.

Main body: We think the expression of TNFR2 confers a level of immunomodulatory properties to its expressing cell and this could be crucially important, particularly, for stem/progenitor and tumor cells. This idea has been already proven in many TNFR2 ${ }^{+}$cells. Different immunosuppressive cells like T regs, regulatory B cells (B regs), myeloid-derived suppressor cells (MDSCs), mesenchymal stem cells (MSCs) and endothelial progenitor cells (EPCs) express TNFR2 and are able to suppress immune cells in presence of TNFa. The other category of rare cells that express TNFR2 is neural cells (NCs). Although little is known about the immunological function of these latter cells, few studies showed their progenitors are able to suppress $T$ cells. Therefore, we hypothesize that the immunosuppressive effect of neural stem cells (NSCs) is potentially TNFa-TNFR2 dependent.
\end{abstract}

Conclusions: NSCs are among the rare cells that express TNFR2 marker and are able to supress T cells. We believe TNFa-TNFR2 immune checkpoint signaling pathway could be responsible for this immunosuppressive effect.

Keywords: TNF-TNFR2 signaling pathway, Immune checkpoint, Immunosuppression, Immunoregulation, Neural progenitor cells, Neural stem cells

\footnotetext{
*Correspondence: sina.naserian@inserm.fr; sina.naserian@cellmedex.com

${ }^{1}$ INSERM UMR-S-MD 1197, Hôpital Paul Brousse, Villejuif, France

${ }^{2}$ CellMedEx, Saint Maur Des Fossés, France

Full list of author information is available at the end of the article
}

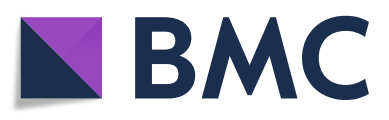

( ) The Author(s). 2020 Open Access This article is licensed under a Creative Commons Attribution 4.0 International License, which permits use, sharing, adaptation, distribution and reproduction in any medium or format, as long as you give appropriate credit to the original author(s) and the source, provide a link to the Creative Commons licence, and indicate if changes were made. The images or other third party material in this article are included in the article's Creative Commons licence, unless indicated otherwise in a credit line to the material. If material is not included in the article's Creative Commons licence and your intended use is not permitted by statutory regulation or exceeds the permitted use, you will need to obtain permission directly from the copyright holder. To view a copy of this licence, visit http://creativecommons.org/licenses/by/4.0/. The Creative Commons Public Domain Dedication waiver (http://creativecommons.org/publicdomain/zero/1.0/) applies to the data made available in this article, unless otherwise stated in a credit line to the data. 


\section{Background}

Tumor necrosis factor alpha (TNF $\alpha$ ) is a proinflammatory cytokine that could modulate both pro- and anti-inflammatory properties [1]. TNF $\alpha$ interacts with two distinct transmembrane receptors, TNFR1 (CD120a) and TNFR2 (CD120b). TNFR1 is expressed ubiquitously on almost all cells, and its binding to TNF $\alpha$ will lead to apoptosis and eventually cell death. TNFR2, however, is limitedly expressed on certain cells including immune cells, endothelial cells (ECs), MSCs and neural cells (NCs), and its interaction with TNFo leads to proliferation and cell survival [2]. Is has been evidenced that TNF $\alpha$ increases the expression of several pro-angiogenic factors such as vascular endothelial growth factor (VEGF), basic fibroblast growth factor (bFGF), and IL-8 in ECs [3]. Moreover, it has been shown that TNF $\alpha$ is involved in proliferation, neuronal differentiation, and neurogenesis [4]. Indeed, TNF $\alpha$-TNFR2 axis generally supports the protective mechanisms, and inversely, TNF $\alpha$-TNFR1 axis is involved in deleterious mechanisms. For example, it has been shown that TNFR1 mediates myocardiac ischemic injuries and has toxic effects in models of myocardial infarction; however, TNFR2 signaling is protective in infract myocardium, heart ischemic injuries, and aging [5]. Moreover, unlike interaction through TNFR1, TNF $\alpha$-TNFR2 signaling can increase the anti-inflammatory and immunosuppressive mechanisms [6].

\section{Main text}

Observing particular immune and physiological reactions of cells expressing TNFR2 molecule encouraged us to study its role in limited cells that express this marker and interestingly found out that in almost all TNFR2 ${ }^{+}$ cells, there is at least one kind of immunoregulatory function. We and others have clearly shown that many immunosuppressive cells like $\mathrm{T}$ regs, $\mathrm{B}$ reg, and MDSCs express TNFR2, and this is directly related to the efficiency of immunosuppression mostly via modulating the secretion of anti-inflammatory cytokines [6-8].

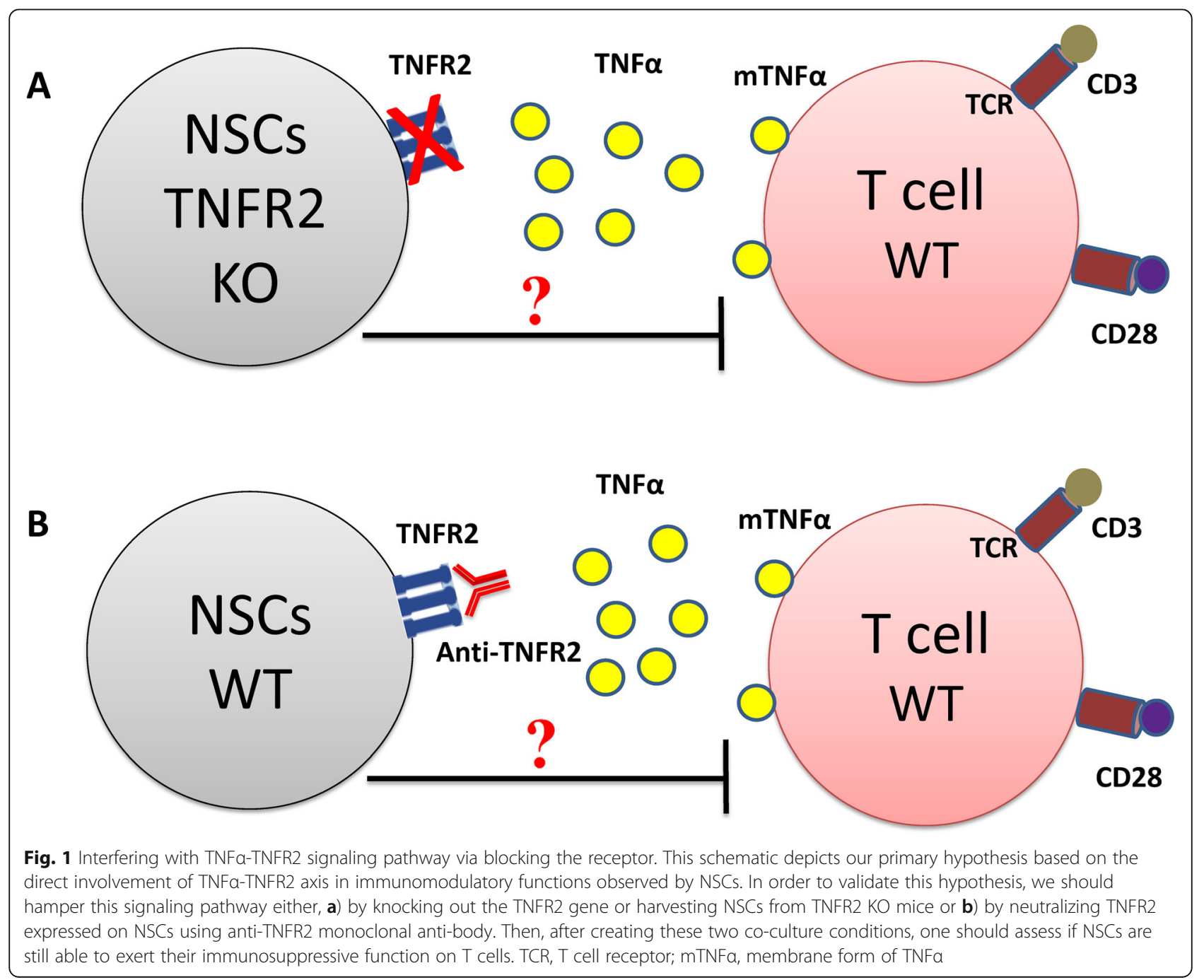




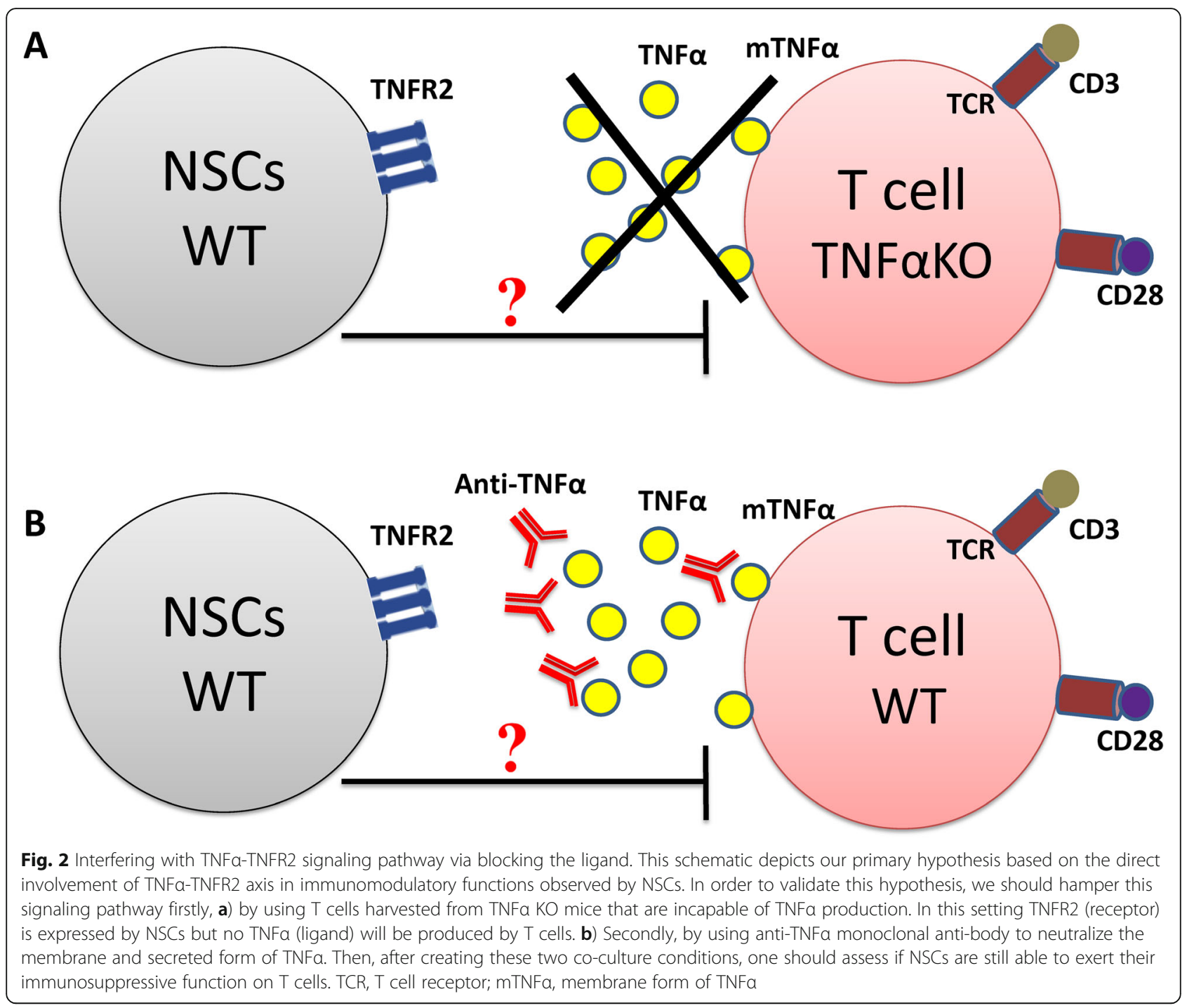

Furthermore, we have been investigating on ECs, particularly, endothelial progenitor cells (EPCs) that are among the rare cells expressing TNFR2 and which TNF $\alpha$ is critically important for their activation, migration, and angiogenic activities. We recently demonstrated that cord blood EPCs (CB-EPCs) and adult peripheral blood EPCs (APB-EPCs) have immunosuppressive and immunomodulatory functions against $\mathrm{T}$ cells. Additionally, we showed that CB-EPCs are able to induce new functional vessels in xenogeneic ischemic mice and are tolerated and resistant in several tissues after their first administration. Very interestingly, our data proved that the EPC immunosuppressive effect was entirely TNF $\alpha$-TNFR2 dependent $[9,10]$.

Likewise, we have shown in several in vitro and in vivo studies that MSCs strongly suppress immune cells in innate and adaptive immune systems. MSCs inhibit effector $\mathrm{T}$ cell ( $\mathrm{T}$ eff) proliferation and function in all autologous, allogenic, and xenogeneic conditions while promoting and inducing $\mathrm{T}$ regs (iT regs). In consequence, they decrease the secretion of pro-inflammatory cytokine [11]. MSC immunoregulatory function can be changed when exposed to an inflammatory microenvironment. TNF $\alpha$ and other inflammatory cytokines have been shown to actively prime MSCs towards more immunosuppressive phenotypes [12]. We have recently investigated the involvement of the TNF $\alpha$-TNFR2 signaling pathway in the immunosuppressive effect of MSCs. Our results demonstrated for the first time that the TNF $\alpha$-TNFR2 signaling pathway plays a critical role in MSC immunomodulatory effect. Unlike WTMSCs, TNFR2 KO-MSCs were remarkably less able to suppress $\mathrm{T}$ cells, down-modulate $\mathrm{T}$ cell activation markers and pro-inflammatory cytokine secretion, and induce active $\mathrm{T}$ regs [13].

The other rare cells that express TNFR2 is neural cells (NCs). It has been shown that TNF $\alpha$ signaling has also 
both positive and negative effects on neurogenesis and is required to moderate the negative impact of cranial irradiation on hippocampal neurogenesis and neuroinflammation [14]. Very interestingly, it has been reported that neural stem/progenitor cells (NSCs) from mouse, rat, and pig are demonstrating immunosuppressive effect against anti-CD3/CD28 polyclonal activated effector T cells [15].

Based on solid results that correlate the expression of TNFR2 and immunoregulatory function, we hypothesize that the immunosuppressive function of NSCs could be also TNFo-TNFR2 dependent. Thus, we think it is necessary to answer this question by neuroscience experts. In order to do that, we suggest co-culturing different NCs including NSCs with different immune cells including $\mathrm{T}$ cells $\left(\mathrm{CD} 4^{+}\right.$and $\mathrm{CD}^{+}$populations) and eventually macrophages and NK cells and to observe if the immunosuppressive effect observed by NSCs is partially or entirely TNFR2 dependent.

In order to prove that, the TNF $\alpha$-TNFR2 signaling pathway must be interfered either by knocking out TNFR2 gene in neural cells or using NSCs harvested from TNFR2 KO mice (Fig. 1a) or using anti-TNFR2 monoclonal antibody (anti-TNFR2-mAb) to block the receptor (Fig. 1b). This approach should be reinforced by using $\mathrm{T}$ cells derived from TNF $\alpha \mathrm{KO}$ mice (Fig. 2a) or application of anti$\mathrm{TNF} \alpha \mathrm{mAb}$ to block the ligand (Fig. $2 \mathrm{~b}$ ). In addition, it will be very important to assess if the immunosuppressive effect demonstrated by NSCs is correlated to their stemness features and the passage/age (population doubling level) of the cells or even mature NCs are able to suppress immune cells through TNF $\alpha$-TNFR2 axis.

In some cancers, the number of neurons is increased, which suggests that a potential process of neurogenesis occurs and this phenomenon could support tumor development and progression. Answering these questions is crucial since administration of anti-TNFR2 agents (TNFR2 antagonist) seems to be an effective way in cancer immunotherapy. Therefore, it is very important to understand which cells are directly affected by this kind of treatment and what will be the consequences in every type of TNFR2 expressing cells.

\section{Conclusions}

Through different in vitro and in vivo experimental approaches, TNF $\alpha$-TNFR2 immune checkpoint signaling pathway was reported important for controlling the immunoregulatroy functions of almost all $\mathrm{TNFR}^{+}$cells including stem/progenitor cells. This axis can modulate different immunological aspects of stem cells such as the production of different anti-inflammatory cytokines. We strongly believe that targeting TNFR2 using its proper antagonist is an effective way to treat cancer as it efficiently controls immunosuppression, tumor angiogenesis, tumor neurogenesis, and survival. Therefore, it is critical to understand what will be the exact effect of anti-TNFR2 treatment in every cell expressing this immunoregulatory marker. NSCs are one of those TNFR2 expressing cells that have not been sufficiently studied for their immunomodulatory features. We hypothesize that there might be a direct relationship between the expression of TNFR2 and the immunosuppressive effect of NSCs.

\section{Abbreviations}

APB: Adult peripheral blood; B reg: Regulatory B cell; bFGF: Basic fibroblast growth factor; CB: Cord blood; CD: Cluster of differentiation; ECs: Endothelial cells; EPCs: Endothelial progenitor cells; IL-8: Interleukin-8; mAb: Monoclonal anti body; MDCSs: Myeloid-derived suppressive cells; MSCs: Mesenchymal stem cells; NCs: Neural cells; NSCs: Neural stem cells; T eff: Effector T cell; T reg: Regulatory T cell; TNFR1: Tumor necrosis factor receptor 1; TNFR2 KO: Tumor necrosis factor receptor 2 knock-out; TNFR2: Tumor necrosis factor receptor 2; TNFa: Tumor necrosis factor alpha; VEGF: Vascular endothelial growth factor

\section{Acknowledgements}

Not applicable

Authors' contributions

S.SH and S. N conceived the hypothesis and wrote the manuscript. G. U read and revised the manuscript. All authors read and approved the final manuscript.

\section{Funding}

Not applicable

Availability of data and materials

Not applicable

Ethics approval and consent to participate

Not applicable

\section{Consent for publication}

Not applicable

\section{Competing interests}

The authors declare that they have no competing interests.

\section{Author details}

${ }^{1}$ INSERM UMR-S-MD 1197, Hôpital Paul Brousse, Villejuif, France. ${ }^{2}$ CellMedEx, Saint Maur Des Fossés, France. ${ }^{3}$ Paris-Saclay University, Villejuif, France.

Received: 3 February 2020 Revised: 21 June 2020

Accepted: 7 July 2020 Published online: 22 July 2020

\section{References}

1. Yang S, Wang J, Brand DD, Zheng SG. Role of TNF-TNF receptor 2 signal in regulatory T cells and its therapeutic implications. Front Immunol. 2018;9: 784. https://doi.org/10.3389/fimmu.2018.00784.

2. Faustman DL, Davis M. TNF receptor 2 and disease: autoimmunity and regenerative medicine. Front Immunol. 2013;4:478. https://doi.org/10.3389/ fimmu.2013.00478.

3. Hoefer IE, van Royen N, Rectenwald JE, et al. Direct evidence for tumor necrosis factor-alpha signaling in arteriogenesis. Circulation. 2002;105(14): 1639-41. https://doi.org/10.1161/01.cir.0000014987.32865.8e.

4. Bernardino L, Agasse F, Silva B, Ferreira R, Grade S, Malva JO. Tumor necrosis factor-alpha modulates survival, proliferation, and neuronal differentiation in neonatal subventricular zone cell cultures. Stem Cells Dayt Ohio. 2008;26(9): 2361-71. https://doi.org/10.1634/stemcells.2007-0914.

5. Monden Y, Kubota T, Inoue T, et al. Tumor necrosis factor-al pha is toxic via receptor 1 and protective via receptor 2 in a murine model of myocardial infarction. Am J Physiol Heart Circ Physiol. 2007;293(1):H743-53. https://doi. org/10.1152/ajpheart.00166.2007.

6. Leclerc M, Naserian S, Pilon C, et al. Control of GVHD by regulatory T cells depends on TNF produced by T cells and TNFR2 expressed by regulatory T cells. Blood. 2016;128(12):1651-9. https://doi.org/10.1182/blood-2016-02700849 . 
7. Ticha $\mathrm{O}$, Moos L, Wajant $\mathrm{H}$, Bekeredjian-Ding I. Expression of tumor necrosis factor receptor 2 characterizes TLR9-driven formation of interleukin-10producing B cells. Front Immunol. 2017;8:1951. https://doi.org/10.3389/ fimmu.2017.01951.

8. Polz J, Remke A, Weber $\mathrm{S}$, et al. Myeloid suppressor cells require membrane TNFR2 expression for suppressive activity. Immun Inflamm Dis. 2014;2(2): 121-30. https://doi.org/10.1002/iid3.19.

9. Naserian S, Abdelgawad ME, Lachaux J, et al. Development of bio-artificial micro-vessels with immunosuppressive capacities: a hope for future transplantations and organoids. Blood. 2019;134(Supplement_1):3610. https://doi.org/10.1182/blood-2019-121395.

10. Naserian S, Abdelgawad ME, Afshar Bakshloo M, et al. The TNF/TNFR2 signaling pathway is a key regulatory factor in endothelial progenitor cell immunosuppressive effect. Cell Commun Signal CCS. 2020;18(1):94. https:// doi.org/10.1186/s12964-020-00564-3.

11. Khosravi M, Bidmeshkipour A, Cohen $\mathrm{J}$, et al. Induction of CD4+CD25+ FOXP3+ regulatory $T$ cells by mesenchymal stem cells is associated with modulation of ubiquitination factors and TSDR demethylation. Stem Cell Res Ther. 2018;9(1):273. https://doi.org/10.1186/s13287-018-0991-1.

12. Yan $L$, Zheng $D, X u R-H$. Critical role of tumor necrosis factor signaling in mesenchymal stem cell-based therapy for autoimmune and inflammatory diseases. Front Immunol. 2018;9:1658. https://doi.org/10.3389/fimmu.2018. 01658.

13. Beldi G, Khosravi M, Abdelgawad ME, et al. TNFa/TNFR2 signaling pathway: an active immune checkpoint for mesenchymal stem cell immunoregulatory function. Stem Cell Res Ther. 2020;11:281. https://doi.org/ 10.1186/s13287-020-01740-5.

14. Chen Z, Palmer TD. Differential roles of TNFR1 and TNFR2 signaling in adult hippocampal neurogenesis. Brain Behav Immun. 2013;30:45-53. https://doi. org/10.1016/j.bbi.2013.01.083.

15. Bonnamain V, Neveu I, Naveilhan P. In vitro analyses of the immunosuppressive properties of neural stem/progenitor cells using antiCD3/CD28-activated T cells. Methods Mol Biol. 2011;677:233-43. https://doi. org/10.1007/978-1-60761-869-0_17.

\section{Publisher's Note}

Springer Nature remains neutral with regard to jurisdictional claims in published maps and institutional affiliations. 